\title{
Rheological Parameters Assessment in Serum, Plasma and Whole Blood of Rats after Administration of Gold Nanoparticles of Different Sizes: In vivo
}

\section{Mohamed Anwar K Abdelhalim ${ }^{1 *}$ and Mohsen Mahmoud Mady ${ }^{1,2}$}

${ }^{1}$ Department of Physics and Astronomy, College of Science, King Saud University, Saudi Arabia

${ }^{2}$ Department of Biophysics, Cairo University, 12613 Giza, Egypt

\begin{abstract}
Background: The evaluation of blood rheology has been underutilised in clinical practice. We performed an array of rheological parameters measurements to quantify the responses of rat plasma, serum and whole blood to gold nanoparticles (GNPs) of different sizes.

Methods: GNPs of various sizes were used in this study. Doses of $0.05 \mathrm{ml}$ of the GNPs were administered to the animals via intraperitoneal injection for a period of 3 days. Blood samples with volumes of nearly $2 \mathrm{ml}$ were obtained from each rat. Various rheological parameters, such as \%torque, shear stress (SS), shear rate (SR), viscosity, plastic velocity, yield stress, consistency index and flow index, were measured in rat plasma, serum and whole blood.

Results: The relationship between SS and SR for rat serum, plasma and whole blood showed linear behaviour with the 10, 20 and $50 \mathrm{~nm}$ GNPs. The viscosities of rat serum, plasma and whole blood with GNPs were decreased with increasing the SR and showed non-linear behaviour. The viscosity of blood serum and plasma was measured at a range of shear rates from 200 to $1375 \mathrm{~s}^{-1}$, while the viscosity of whole blood was measured at 75 to $600 \mathrm{~s}^{-1}$.

Conclusions: The GNP size has a considerable influence on the various rheological parameters for rat blood at a fixed temperature of $37^{\circ} \mathrm{C}$. The decrease in viscosity of $50 \mathrm{~nm}$ GNPs compared to 10 and $20 \mathrm{~nm}$ GNPs may be attributed to decrease in number of NPs and GNP surface area. It can be concluded that the GNPs probably cause erythrocyte deformability, and their interactions with blood proteins may cause a decrease in serum, plasma and whole blood viscosities under a given level of applied SS and SR compared to the control. This study suggests that further experimental work taking nanoparticle surface properties into consideration should be performed.
\end{abstract}

Keywords: Gold nanoparticle sizes; Serum; Plasma; Whole blood; Rheological parameters; Rats

\section{Introduction}

Hemorheology is the study of the flow properties of blood and its elements (plasma and formed elements, including erythrocytes, white blood cells and platelets). There is increasing evidence that the flow properties of blood are among the main determinants of proper tissue perfusion, and alterations in these properties play significant roles in disease processes; therefore, knowledge of these properties is vital to our understanding of Hemorheology [1].

Blood viscosity is determined by plasma viscosity, haematocrit (volume fraction of erythrocytes, which constitutes $99.9 \%$ of the cellular elements) and the mechanical behaviour of erythrocytes. Therefore, erythrocyte mechanics represent the major determinant of the flow properties of blood. Erythrocytes have unique mechanical behaviours that can be discussed in terms of erythrocyte deformability and aggregation [2].

Whole-blood viscosity is a predictor of stroke, carotid intima-media thickening, and carotid atherosclerosis. However, in most studies, whole blood viscosity was measured at a few non-specific shear rates, and these data do not reflect the complete rheological characteristics found in these studies [3].

Blood is a unique fluid. It exhibits non-Newtonian characteristics, and its viscosity is dependent on the shear rate. The major determinants of whole-blood viscosity are hematocrit, plasma viscosity, and red cell aggregation and deformation under conditions of low and high shear rates [4-6].

In hyperviscosity syndromes, plasma viscosity is a better indicator in follow-up examinations. In rheumatoid arthritis, sensitivity and specificity of plasma viscosity are better than that of C-reactive protein. The plasma fibrinogen concentration and plasma viscosity are elevated in unstable angina pectoris and stroke, and their higher values are associated with higher rates of major adverse clinical events. The elevation of plasma viscosity is correlated with the progression of coronary and peripheral artery diseases. Thus, plasma viscosity should be measured routinely in medical practice [7].

Nanoparticle studies are becoming much more common owing to their novel physical and chemical attributes in biological and medical applications [8-11].

The Surface Plasmon Resonance (SPR) property of NPs allows the use of GNPs in many biological and medical applications. GNPs are used as immunostaining marker particles for electron microscopy and as chromophores for immunoreactions and nucleic acid hybridisation $[12,13]$. Their application for gene delivery into cells has also been reported [14-17]. In addition, GNPs have attracted attention as photothermal agents in hyperthermia treatment [18].

The sizes of the NPs are similar to the sizes of most biological molecules. For this reason, the GNPs can be used for both in vivo and in

*Corresponding author: Mohamed Anwar K. Abdelhalim, Associate Professor, Department of Physics and Astronomy, College of Science, King Saud University, P.O. 2455, Riyadh 11451, Saudi Arabia, E-mail: abdelhalimmak@yahoo.com

Received May 25, 2012; Accepted July 16, 2012; Published July 18, 2012

Citation: Abdelhalim MAK, Mady MM (2012) Rheological Parameters Assessment in Serum, Plasma and Whole Blood of Rats after Administration of Gold Nanoparticles of Different Sizes: In vivo. J Nanomed Nanotechol 3:145. doi:10.4172/2157-7439.1000145

Copyright: (c) 2012 Abdelhalim MAK, et al. This is an open-access article distributed under the terms of the Creative Commons Attribution License, which permits unrestricted use, distribution, and reproduction in any medium, provided the original author and source are credited. 
vitro biomedical applications. Therefore, increased attention has been placed on the applications of NPs in biology and medicine.

Viscoelastic properties can be applied to the quality control of raw materials, final products, and manufacturing processes. Furthermore, the release of a drug from a semi-solid carrier is influenced by the carrier's rheological behaviour. The effect of certain parameters, such as the storage time and temperature, on the quality of the GNPs as pharmaceutical products can be investigated via rheological measurements. Rheological analysis can be used as a sensitive tool in predicting the physical properties of the GNPs of different sizes.

The objective of this study was to examine the effects of the daily intraperitoneal administration of $0.05 \mathrm{ml}$ of the GNPs of different sizes $(10,20$ and $50 \mathrm{~nm})$ for 3 days on various rheological parameters in rat serum, plasma and whole blood over a wide range of shear rates.

\section{Materials and Methods}

\section{GNP sizes}

GNPs (in sizes of the 10, 20 and $50 \mathrm{~nm}$ ) were purchased (Product $\mathrm{MKN}-\mathrm{Au}$ ) and used in this study. The GNPs were in aqueous solution (10 mm GNPs: Product MKN-Au-010, concentration 0.01\% Au; $20 \mathrm{~nm}$ GNPs: Product MKN-Au-020, concentration 0.01\% Au; $50 \mathrm{~nm}$ GNPs: Product MKN-Au-050, concentration $0.01 \% \mathrm{Au}$ ).

\section{Animals}

Healthy male Wistar-Kyoto rats (8-12 weeks old; approximately $250 \mathrm{~g}$ body weight) that were obtained from the Laboratory Animal Centre (LAC) (College of Pharmacy, King Saud University) were housed in pairs in humidity- and temperature-controlled ventilated cages on a $12 \mathrm{~h}$ day/night cycle. A rodent diet and water were provided. Fifty rats were individually caged and divided into the control group (NG: $\mathrm{n}=8$ ), group 1 (A: infusion of the $20 \mathrm{~nm}$ GNPs for 3 days; $\mathrm{n}=6$ ), group 2 (A: infusion of the $10 \mathrm{~nm}$ GNPs for 3 days; $n=6$ ) and group 3 (A: infusion of the $50 \mathrm{~nm}$ GNPs for 3 days; $\mathrm{n}=6$ ). All experiments were conducted in accordance with the guidelines approved by King Saud University Local Animal Care and Use Committee.

\section{GNPs administration and preparation of serum, plasma and whole blood}

Doses of $0.05 \mathrm{ml}$ of the GNPs $(10,20$ and $50 \mathrm{~nm})$ in aqueous solutions were administered to the animals via intraperitoneal administration for a period of 3 days. The rats were anesthetised by inhalation of $5 \%$ isoflurane until muscular tonus relaxed. Blood and several organs (liver, heart, lung and kidney) were collected from each rat.

Blood samples of nearly $2 \mathrm{ml}$ were collected into three polypropylene tubes: the $1^{\text {st }}$ tube for serum, the $2^{\text {nd }}$ tube for plasma and the $3^{\text {rd }}$ tube for whole blood. The serum was prepared by allowing the blood to clot at $37^{\circ} \mathrm{C}$. The blood was then centrifuged at 3,000 rpm for ten minutes. The blood for plasma was collected in EDTA. Whole blood was prepared by adding $0.8 \mathrm{ml}$ of heparin to $0.8 \mathrm{ml}$ of collected blood.

\section{Experimental setup and rheological parameters}

The following experimental setup was used to measure several rheological parameters in rat serum, plasma and whole blood after the administration of the 10, 20 and $50 \mathrm{~nm}$ GNPs. The rheological parameters tested were viscosity, \%torque, Shear Stress (SS), Shear Rate (SR), plastic viscosity, yield stress, and consistency index. These parameters were measured using a Brookfield LVDV-III Programmable rheometer (cone-plate viscometer; Brookfield Engineering Laboratory, Incorporation, Middleboro, USA) that was supplied with a temperature bath controlled by a computer. The rheometer was guaranteed to be accurate within $\pm 1 \%$ of the full scale range of the spindle/speed combination, and in-use reproducibility is within $\pm 0.2 \%$.

The rheological parameters were measured at $37^{\circ} \mathrm{C}$. The temperature inside the sample chamber was carefully monitored using a temperature sensor during the viscosity measurements.

A cone and plate sensor with a diameter of $2.4 \mathrm{~cm}$ and an angle of 0.8 was used. The rheometer was calibrated using standard fluids. This viscometer has a viscosity measurement range of 1.5-30,000 $\mathrm{mPa}$ s.

The spindle type (SC-40) and its speed combinations produce results with high accuracy when the applied torque is within the range of $10-100 \%$; the appropriate spindle was chosen accordingly.

An aqueous solution of $0.5 \mathrm{ml}$ of each size of GNP was poured into the sample chamber of the rheometer. The spindle was immersed and rotated in these gold nanofluids in a speed range of 20 to 180 RPM in 20 minutes. The viscous drag of the GNP aqueous solution against the spindle was measured by the deflection of the calibrated spring.

\section{Statistical analysis}

The results of this study were expressed as mean \pm standard deviation (Mean $\pm \mathrm{SD}$ ). To assess the significance of the differences between the control group and the 3 experimental groups (G1A, G2A and G3A), a statistical analysis was performed using one-way analysis of variance (ANOVA) for repeated measurements, with significance assessed at the $5 \%$ confidence level.

\section{Results and Discussions}

\section{Rheological parameters measurements}

This study is unique in examining the relationships between various rheological parameters in rat plasma, serum and whole blood after the administration of $0.05 \mathrm{ml}$ of the GNPs of various sizes at a fixed temperature of $37^{\circ} \mathrm{C}$ and a wide range of shear rates using a Brookfield LVDV-III Programmable rheometer.

The data in the present study suggest that at these shear rates, serum, plasma and whole blood viscosities are influenced by the size and shape of the administered GNPs, the number of NPs and the GNP surface area.

The viscosities of blood serum and plasma were measured at a shear rate range of 200 to $1375 \mathrm{~s}^{-1}$, while the viscosity of whole blood was measured at a shear rate range of 75 to $600 \mathrm{~s}^{-1}$. The shear stress (SS) and shear rate (SR) of G1A $(20 \mathrm{~nm}), \mathrm{G} 2 \mathrm{~A}(10 \mathrm{~nm})$ and G3A $(50 \mathrm{~nm})$ for plasma, serum and whole blood were linearly related as shown in Figures 1, 3 and 5, and the SS and SR relationships of serum, plasma and whole blood can be written as shown in Table 1.

The viscosities of rat serum, plasma and whole blood with the 50 $\mathrm{nm}$ GNPs were significantly lower than those with the $10 \mathrm{~nm}$ GNPs. The decrease in viscosity of the $50 \mathrm{~nm}$ GNP solutions may be attributed to the decrease in the number of GNPs and decreased GNP surface area. For all of the GNPs, the highest viscosity values were observed for whole blood compared to plasma and serum as shown in Figures 2,4 and 6 .

The viscosities of rat serum, plasma and whole blood with the10, 20 and $50 \mathrm{~nm}$ GNPs were decreased with increasing the SR. The viscosity 


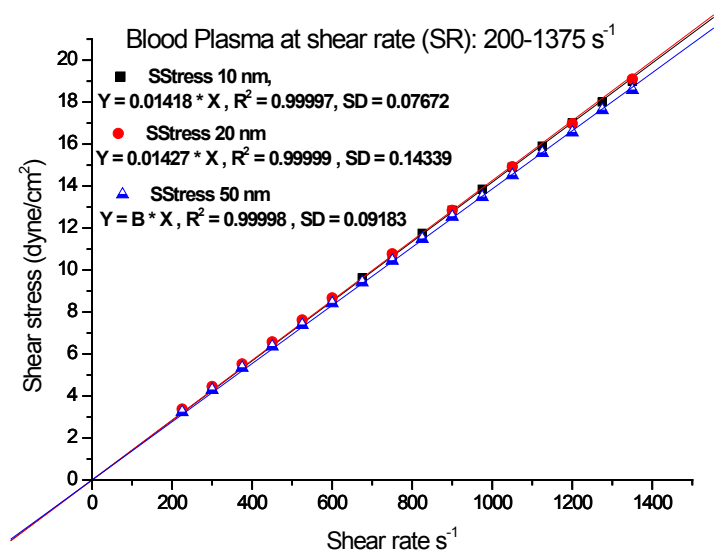

Figure 1: The relationship between shear stress and shear rate for blood plasma of rat at shear rate range of $200-1375 \mathrm{~s}^{-1}$.

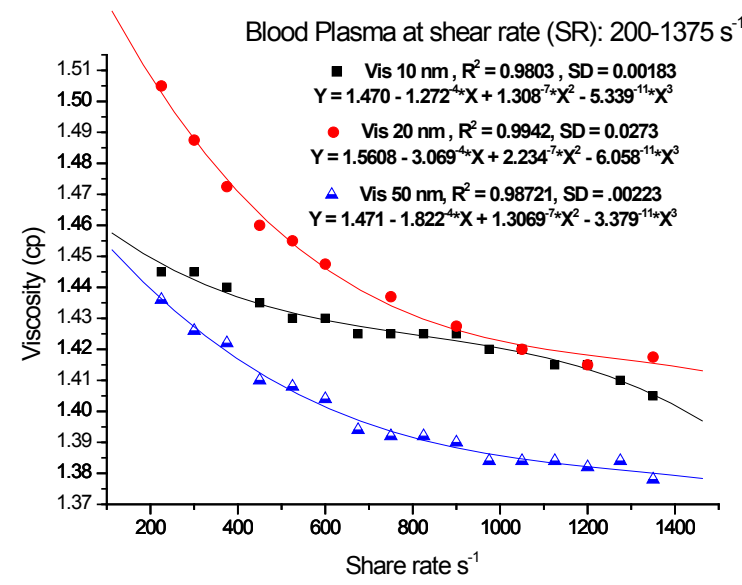

Figure 2: The relationship between viscosity and shear rate for blood plasma of rat at shear rate range of $200-1375 \mathrm{~s}^{-1}$.

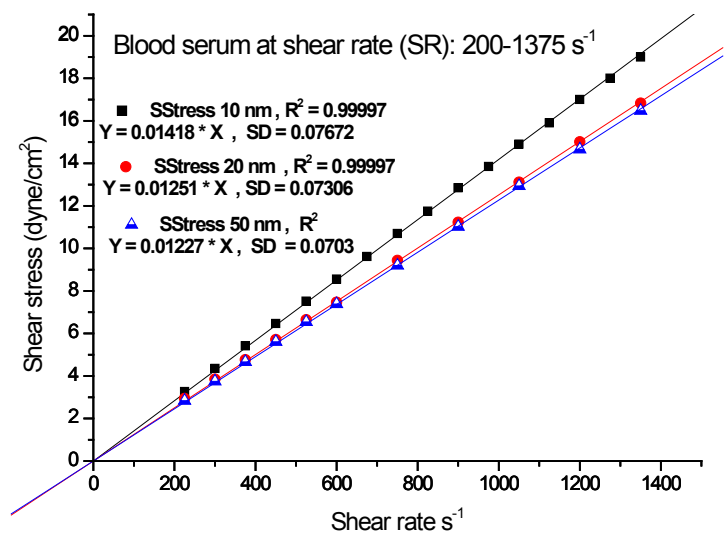

Figure 3: The relationship between shear stress and shear rate for blood serum of rat at shear rate range of $200-1375 \mathrm{~s}^{-1}$.

and SR relationships of rat serum, plasma and whole blood with the10, 20 and $50 \mathrm{~nm}$ GNPs were non-linearly related as shown in Figures 2, 4 and 6.
This study suggests that the decrease in serum, plasma and whole blood viscosities with the 10, 20 and $50 \mathrm{~nm}$ GNPs may be attributed to the decrease in haematocrit and cytoplasmic haemoglobin concentration of erythrocytes, erythrocyte deformability (degree of

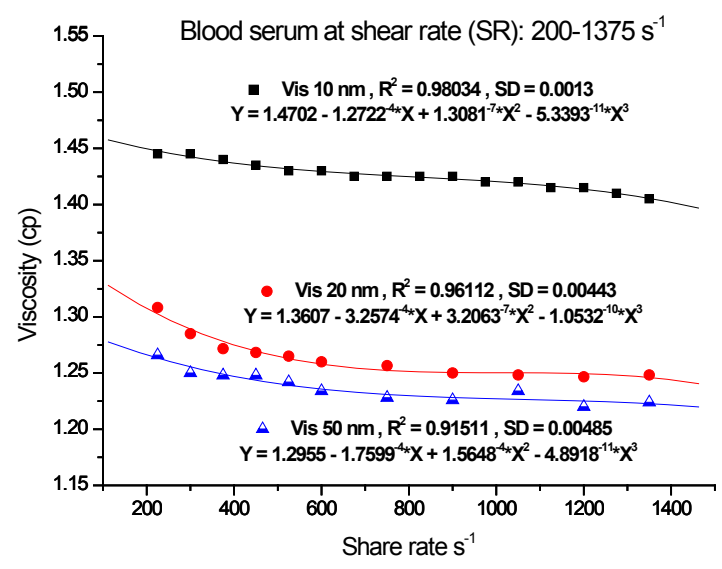

Figure 4: The relationship between viscosity and shear rate for blood serum of rat at shear rate range of $200-1375 \mathrm{~s}^{-1}$.

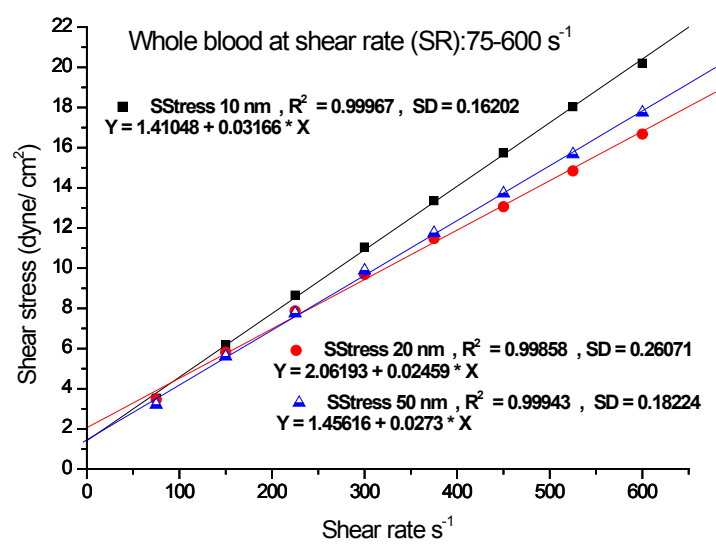

Figure 5: The relationship between shear stress and shear rate for whole blood of rat at shear rate range of $75-600 \mathrm{~s}^{-1}$.

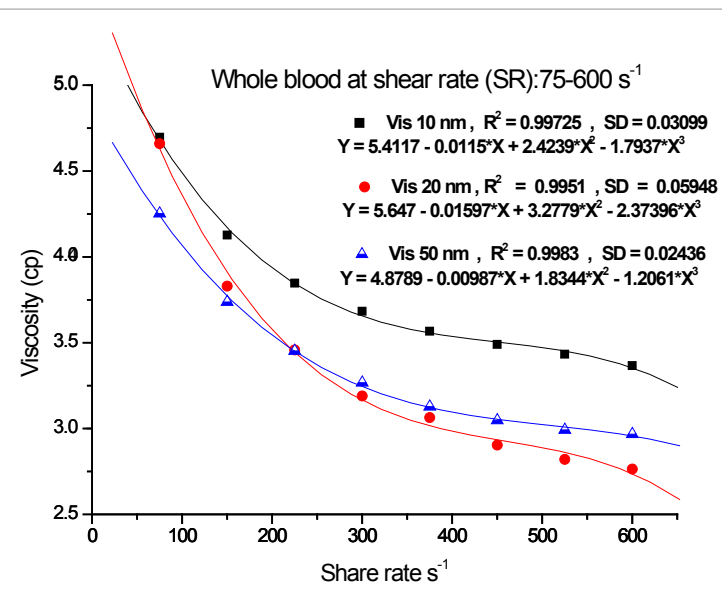

Figure 6: The relationship between viscosity and shear rate for whole blood of rat at shear rate range of $75-600 \mathrm{~s}^{-1}$. 
Citation: Abdelhalim MAK, Mady MM (2012) Rheological Parameters Assessment in Serum, Plasma and Whole Blood of Rats after Administration of Gold Nanoparticles of Different Sizes: In vivo. J Nanomed Nanotechol 3:145. doi:10.4172/2157-7439.1000145

Page 4 of 5

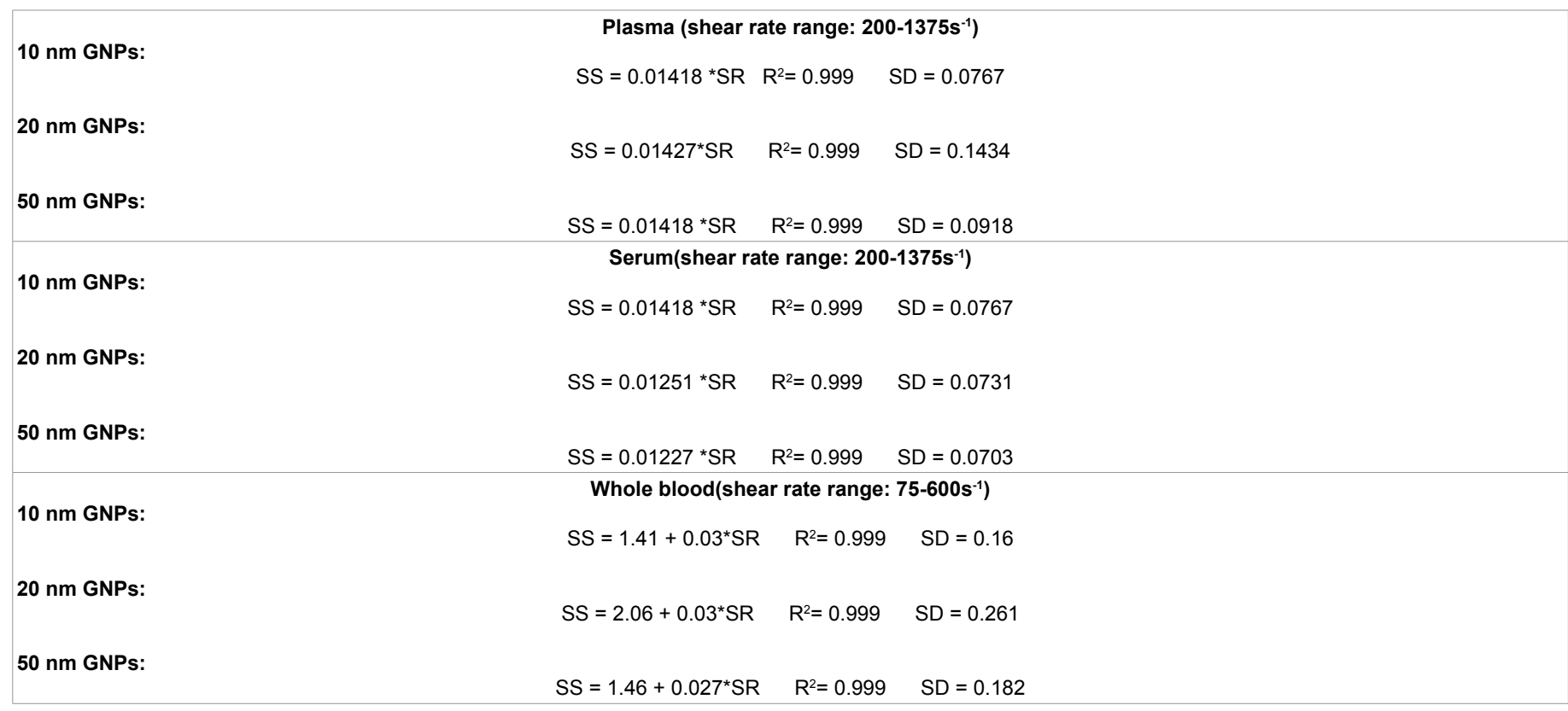

Table 1: The relationship between shear stress and shear rate for plasma, serum and whole blood of rat at wide range of shear rate.

shape change under a given level of applied shear stress and shear rate), and the changes induced in the viscoelastic properties of erythrocyte membranes.

Plasma viscosity is determined by the water content and macromolecular components of plasma, so the factors that affect blood viscosity are the plasma protein concentration and the types of proteins present in the plasma. However, these effects are minor compared to the effect of hematocrit, so they are effectively insignificant. An elevation of plasma viscosity correlates with the progression of coronary and peripheral vascular diseases. Anaemia can lead to decreased blood viscosity, which may lead to heart failure [19].

The shape change of erythrocytes under applied forces is reversible, and the biconcave-discoid shape, which is normal for most mammals, is maintained after the removal of the deforming forces. In other words, erythrocytes behave like elastic bodies, but they also resist shape change under the influence of deforming forces. This viscoelastic behaviour of erythrocytes is determined by the following three properties: 1) their biconcave-discoid geometric shape provides extra surface area for the cell, enabling shape changes without increasing the surface area; this type of shape change requires significantly smaller forces than those required for shape changes with surface area expansion; 2) cytoplasmic viscosity, which reflects the cytoplasmic haemoglobin concentration of erythrocytes; and 3) the viscoelastic properties of the erythrocyte membrane, which are mainly determined by their special membrane skeletal network [20].

The effect of a protein on plasma viscosity depends on its molecular weight and structure. A less spheroid shape, higher molecular weight, higher aggregating capacity, and higher temperature or $\mathrm{pH}$ sensitivity of a protein will lead to higher plasma viscosity [7]. Plasma is a Newtonian fluid, and its viscosity does not depend on flow characteristics. Its normal value is $1.10-1.30 \mathrm{mPa} s$ at $37^{\circ} \mathrm{C}$, which is independent of age and gender [7]. The measurement has high stability and accuracy, so the detection of small alterations may be pathologically important. Inflammation and tissue injuries resulting in changes in plasma proteins can increase its value with high sensitivity but low specificity [19-21].
GNPs strongly associate with essential blood proteins, where the binding constant and degree of cooperatively of particle-protein binding depends on the particle size and the native protein structure. Moreover, the thickness of the adsorbed protein layer (bare NP diameter $<50 \mathrm{~nm}$ ) progressively increases with the NP size, which causes effects that have potential importance for understanding the general NP aggregation in biological media and the interactions of NP with biological materials [21].

In this manuscript the author used GNPs with three different sizes that are available commercially as models for rheological study. However, it is not clear that the surface properties of the GNPs are critically important influencing the interaction with the proteins in the blood. Illustration on the surface properties would help to gain deeper insight into interactions between GNPs and blood, thus the rheological behaviour of blood and GNPs is relevant. Therefore, this study suggests that further experimental work taking nanoparticle surface properties into consideration should be performed.

\section{Conclusions}

This study indicates that GNP size has a considerable influence on the various rheological parameters measured in rat serum, plasma and whole blood at $37^{\circ} \mathrm{C}$.

The decrease in viscosity of blood fluids in the presence of the 50 nm GNPs compared to the 10 and $20 \mathrm{~nm}$ GNPs may be attributed to the decrease in the number of GNPs and the decreased GNP surface area.

The viscosities of rat serum, plasma and whole blood with the10, 20 and $50 \mathrm{~nm}$ GNPs were decreased with increasing the SR. The viscosity and SR relationships of rat serum, plasma and whole blood with the10, 20 and $50 \mathrm{~nm}$ GNPs showed non-linear behaviour. For all of the GNPs, the highest viscosity values were observed in whole blood compared to plasma and serum.

Based on the present results of erythrocyte rheological properties, it can be concluded that the GNPs would probably cause erythrocyte deformability. 
Citation: Abdelhalim MAK, Mady MM (2012) Rheological Parameters Assessment in Serum, Plasma and Whole Blood of Rats after Administration of Gold Nanoparticles of Different Sizes: In vivo. J Nanomed Nanotechol 3:145. doi:10.4172/2157-7439.1000145

\section{Authors' Contributions}

AMAK analysed data and interpreted and wrote the final draft of this manuscript. The animal model used in this study was obtained from the Laboratory Animal Centre (College of Pharmacy, King Saud University). AMAK conceived the plan and design and obtained research grants for this study. The authors have read and approved the final manuscript.

\section{Acknowledgements}

The authors are very grateful to NPST. This research was financially supported by the National Science and Technology Innovation Plan (NSTIP), Research No. 08-ADV206-02 and Research No. 09-NAN670-02, College of Science, King Saud University, Saudi Arabia.

\section{References}

1. Baskurt OK (2007) Handbook of hemorheology and hemodynamics. IOS Press, Amsterdam, Netherlands.

2. Baskurt OK, Meiselman HJ (2003) Blood rheology and hemodynamics. Semin Thromb Haemost 29: 435-450.

3. Baskurt OK, Boynard M, Cokelet GR, Connes P, Cooke BM, et al. (2009) New guidelines for hemorheological laboratory techniques. Clin Hemorheol Microcirc 42: 75-97.

4. Koenig W, Ernst E (1992) The possible role of hemorheology in atherothrombogenesis. Atherosclerosis 94: 93-107.

5. Dutta A, Tarbell JM (1996) Influence of non-Newtonian behavior of blood on flow in an elastic artery model. J Biomech Eng 118: 111-119.

6. Goldsmith HL, Turitto VT (1986) Rheological aspects of thrombosis and haemostasis: basic principles and applications. ICTH-Report--subcommittee on rheology of the international committee on thrombosis and haemostasis. Thromb Haemost 55: 415-435.

7. Késmárky G, Kenyeres $P$, Rábai M, Tóth K (2008) Plasma viscosity: a forgotten variable. Clin Hemorheol Microcirc 39: 243-246.

8. Sperling RA, Gil PR, Zhang F, Zanella M, Parak WJ (2008) Biological applications of gold nanoparticles. Chem Soc Rev 37: 1896-1908.

9. Wan H, Chen L, Chen J, Zhou H, Zhang D, et al. (2008) Synthesis and fluorescence property of the gold nanoparticles modified with 9-Methyl-9-(8thiooctyl)-fluorene. J Dispers Sci Technol 29: 999-1002.
10. Tokonami S, Shiigi H, Nagaoka T (2008) Preparation of nanogapped gold nanoparticle array for DNA detection. Electroanalysis 20: 355-360.

11. Huang X, Jain PK, El-Sayed IH, El-Sayed MA (2007) Gold nanoparticles: interesting optical properties and recent applications in cancer diagnostics and therapy. Nanomedicine 2: 681-693.

12. Mirkin CA, Letsinger RL, Mucic RC, Storhoff JJ (1996) A DNA-based method for rationally assembling nanoparticles into macroscopic materials. Nature 382 607-609.

13. Huber M, Wei TF, Muller UR, Lefebvre PA, Marla SS, et al. (2004) Gold nanoparticle probe-based gene expression analysis with unamplified tota human RNA. Nucleic Acids Res 32: e137.

14. Ow Sullivan MM, Green JJ, Przybycien TM (2003) Development of a nove gene delivery scaffold utilizing colloidal gold-polyethylenimine conjugates for DNA condensation. Gene Ther 10: 1882-1890.

15. Sandhu KK, McIntosh CM, Simard JM, Smith SW, Rotello VM (2002) Gold nanoparticle-mediated transfection of mammalian cells. Bioconjug Chem 13 3-6.

16. Niidome T, Nakashima K, Takahashi H, Niidome Y (2004) Preparation of primary amine-modified gold nanoparticles and their transfection ability into cultivated cells. Chem Commun (Camb) 1978-1979.

17. Kawano T, Yamagata M, Takahashi H, Niidome Y, Yamada S, et al. (2006) Stabilizing of plasmid DNA in vivo by PEG-modified cationic gold nanoparticles and the gene expression assisted with electrical pulses. J Control Release 111 382-389.

18. El-Sayed IH, Huang X, El-Sayed MA (2006) Selective laser photo-therma therapy of epithelial carcinoma using anti-EGFR antibody conjugated gold nanoparticles. Cancer Lett 239: 129-135.

19. Tefferi A (2003) A contemporary approach to the diagnosis and management of polycythemia vera. Curr Hematol Rep 2: 237-241.

20. Chien S (1987) Red cell deformability and its relevance to blood flow. Annua Rev Physiol 49: 177-192.

21. Lacerda SH, Park JJ, Meuse C, Pristinski D, Becker ML, et al. (2010) Interaction of gold nanoparticles with common human blood proteins. ACS Nano 4: 365 379 . 